\title{
Modelling the temperature in joint friction of industrial manipulators
}

\author{
Luca Simoni $^{a}$, Manuel Beschi ${ }^{b}$, Giovanni Legnani ${ }^{a}$, Antonio Visioli ${ }^{a *}$ \\ ${ }^{a}$ Dipartimento di Ingegneria Meccanica e Industriale \\ University of Brescia, Via Branze 38, I-25123 Brescia, Italy \\ ${ }^{b}$ Istituto di Tecnologie Industriali e Automazione, \\ National Research Council (ITIA-CNR), Via Corti 12, I-20133 Milan, Italy
}

This is the pre-peer reviewed version of the following article: Modelling the temperature in joint friction of industrial manipulators, which has been published in final form at 10.1017/S0263574717000509. This article may be used for non-commercial purposes in accordance with Journal terms and conditions for Self-Archiving.

\begin{abstract}
In this paper, a new model for joint dynamic friction of industrial robot manipulators is presented. In particular, the effects of the temperature in the joints are considered. A polynomial-based model is proposed and the parameter estimation is performed without the need of a joint temperature sensor. The use of an observer is then proposed to compensate for the uncertainty in the initial estimation of the temperature value. A large experimental campaign show that the model, in spite of the simplifying assumptions made, is effective in estimating the joint temperature and therefore the friction torque during the robot operations, even for values of velocities that have not been previously employed.
\end{abstract}

Keywords: Friction, industrial manipulators, modelling and identification.

* Corresponding author.

Email addresses: \{1.simoni002, giovanni.legnani, antonio.visioli\} @ unibs.it (L. Simoni, G. Legnani, A. Visioli), manuel.beschi@ itia.cnr.it (M. Beschi). 


\section{Introduction}

It is well known that friction is one of the major problems that has to be faced in the design of motion control systems. Actually, the compensation of this effect is crucial to improve the performance for industrial robot manipulators, not only when they are employed in free motion tasks (for example by including a suitable feedforward term in the control law), but also for more advanced applications like those involving force control or manual guidance (for example for lead-through programming) [7, 17,29]. In fact, an accurate knowledge of friction effects allows the designer to obtain a more precise estimation of the external forces. In addition, the knowledge of the complete dynamic model of the robot, including friction, allows the user to predict the energy consumption of the manipulator and therefore to optimize tasks in this context. Finally, the accurate knowledge of the complete dynamic model can be used in long-term maintenance activities by analyzing the trend of the model prediction error. However, in general, friction model uncertainties and variations reduce the estimation reliability.

Friction is a complex phenomenon that arises when there is a relative motion between two bodies in contact, or when a body moves inside a fluid. A complete model of friction is very difficult to obtain because friction depends on many factors such as the relative velocity between the bodies in contact, the geometry and the materials of the surfaces, the presence and the type of lubricants, the temperature and so on. For this reason, many friction models have been already proposed in literature in order to capture and compensate for the main components [30]. Indeed, it has been recognized until now that friction force mainly depends on the motion speed. In general, friction models can be divided into static and dynamic ones [3,10]. In static models the relation between relative speed and friction force is, as the name explains, static, while in dynamic models the friction force depends not only on the relative speed of motion, but also on a state variable that is able to take into account the state of the system. Typical friction static models are, for example, the Coulomb model, the viscous, the Stribeck and the polynomial ones [32,35], while the most famous friction dynamic models are the Dahl's one [9,36], the LuGre one [1,2,12,23], the Leuven model [19,31] and the Maxwell-slip model and its extensions [11,20,24,26].

Although these models have been effectively employed in the industrial practice, there is the need to improve them to enhance the capabilities of the industrial robots. In fact, friction force varies with time and this motivates the need of adaptive friction compensation techniques [13,15,27]. Indeed, it has to be recognized that the temperature plays a key role in friction $[21,22,25]$ as friction transforms mechanical energy into heat which causes the temperature to increase [18] and, on the other side, the characteristics of the speed reducers change with the temperature [37]. This issue has been recently investigated thoroughly. In particular, a temperature dependent friction model has been proposed in [4-6], where, however, it is assumed that the joint temperature is measured, which is rather inconvenient in practical cases where it is not normally possible to measure the temperature inside gearboxes.

In this paper, which is an extended version of [28] (note that a similar approach has been presented at the same time in [8]), after showing that friction changes significantly during the robot operations (because of the temperature), we propose a model based on a polynomial function of the joint dynamic friction where the coefficients depends on the temperature estimation obtained by means of a thermal model. The identification of the model parameters that does not employ any additional temperature sensor is described. Then, a standard 
observer is used to compensate for an initial error in the estimation of the environmental temperature. An extensive experimental campaign has been performed in order to verify the correctness of the proposed model and to discuss its applicability in practical cases, when different robot tasks are considered. The main advantage of the proposed method is that the friction function can be determined also for velocities that have not been previously employed in the robot operations. In fact, in this case the use of interpolation method might fail because of the temperature variation. Note that, although static friction is also a significant element that is detrimental for performance when the robot moves at very low velocities [14], in this paper we focus on dynamic friction and therefore on relatively high speed tasks, as the polynomial model is very effective in this case.

The paper is structured as follows. The basic polynomial friction model is described in Section 2, while the improved model that takes into account the temperature effects is proposed in Section 3. The robot used in the experiments of this paper is presented in Section 4. The identification procedure is given in Section 5. The observer based technique is proposed in Section 6 and experimental results are given in Section 7. Concluding remarks are in Section 8.

\section{Polynomial Friction Model}

It is well known that the dynamic model of a robot manipulator can be expressed as

$$
\boldsymbol{\tau}=\mathbf{F}(\theta, \dot{\theta}, \ddot{\theta})+\boldsymbol{\tau}_{\boldsymbol{f}}
$$

where $\boldsymbol{\tau}$ is the vector of the joint torques, $\boldsymbol{\theta}$ is the vector of the joint positions, $\mathbf{F}$ is the function that includes inertial, Coriolis and gravity terms and $\tau_{f}$ is the vector of the friction terms. It is worth stressing that if the robot is characterized by elastic behaviors on links or joints, those effect can be added in function $\mathbf{F}$, without modifications on the mathematical formulation of the proposed friction model. As already mentioned in the introduction, friction is a complex phenomenon that depends on many factors (including temperature, as it will be shown in the next sections) but, most of all, on velocity. For this reason many models that depend only on velocity have been proposed in the literature. Among them, the polynomial friction model has been selected in this paper because it is the model that best fits the experimental friction torque data obtained with the robot manipulator used as experimental setup (see Section 4). Indeed, in the examined cases, a polynomial function of third order is capable to represent quite accurately the dynamic friction effects, without losing linearity in the parameters. Further, it is also able to represent a viscous effect that is not necessarily linear (see Figure 2 in Section 3).

By considering for simplicity a single joint, the friction torque function can be therefore represented as

$$
\tau_{f}=\left[c_{0}+c_{1}|\omega|+c_{2}|\omega|^{2}+c_{3}|\omega|^{3}\right] \operatorname{sgn}(\omega)
$$

where $\omega=\dot{\theta}$ is the measured joint velocity and it has to be noted that a symmetric function (w.r.t. the velocity) is considered, that is, the coefficients $c_{0}, \ldots, c_{3}$ are the same for positive and negative velocities. The polynomial coefficients can be determined easily by moving only one joint of the robot at a time, so that a one degree-of-freedom system can be considered for each joint (see Figure 1). In this case the dynamics equation of the joint can be written as

$$
\tau-\tau_{f}-\tau_{w}=J \dot{\omega}
$$




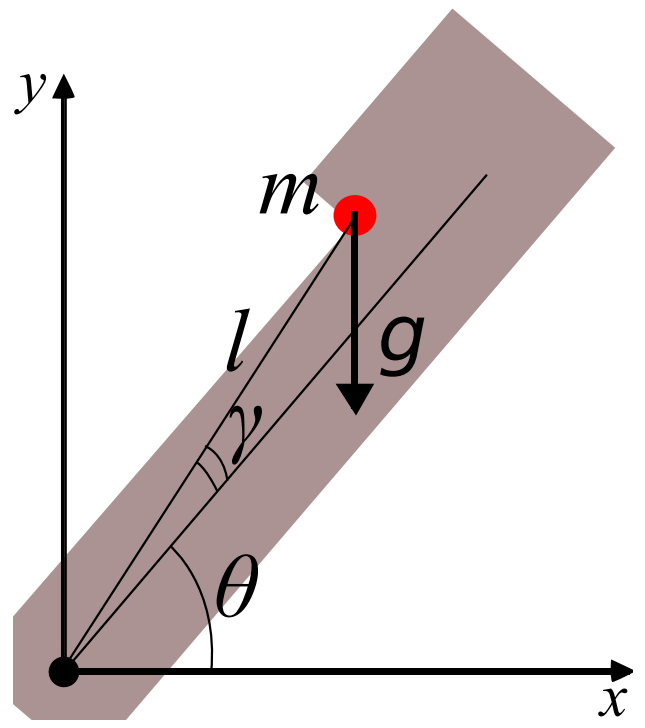

Figure 1: Weight scheme of a single joint robot.

where $J$ is the inertia seen by the motor, $\tau$ is the motor torque, $\tau_{f}$ is the friction torque and $\tau_{w}$ is the torque related to the gravitational force. From (3) it is immediate to write

$$
\tau=J \dot{\omega}+\tau_{f}+\tau_{w}
$$

Now, a standard least-squares method can be applied to estimate the friction torque coefficients collecting the motor torques while performing suitable motion. In fact, (3) can be rewritten by expressing all the terms as:

$$
\begin{aligned}
\tau & =J \dot{\omega}+\tau_{f}+\tau_{w} \\
& =J \dot{\omega}+c_{0} \operatorname{sgn}(\omega)+c_{1} \omega+c_{2} \omega^{2} \operatorname{sgn}(\omega)+c_{3} \omega^{3}+P_{x} \cos (\theta)-P_{y} \sin (\theta)
\end{aligned}
$$

where the gravity compensation torque has been expressed as

$$
\tau_{w}=P_{x} \cos (\theta)-P_{y} \sin (\theta)
$$

with $\theta$ the joint (measured) position and

$$
P_{x}=m g l \cos (\gamma) \quad P_{y}=m g l \sin (\gamma)
$$

where $m$ is the mass of the moving part of the robot, $g$ is the gravity acceleration, $l$ is the distance between the joint axis of rotation and the centre of mass of the moving part of the manipulator and $\gamma$ is the angle of the centre of the mass position when $\theta=0$ (see Figure 1). Thus, (5) can be written in matrix form (where in each row there are all the $n$ samples collected at a given sampling time) as:

$$
\tau=\mathbf{M X}
$$

where

$$
\tau=\left[\begin{array}{llll}
\tau_{1} & \tau_{2} & \ldots & \tau_{n}
\end{array}\right]^{T}
$$


is the vector of the measured motor torques,

$$
\mathbf{M}=\left[\begin{array}{ccccccc}
\dot{\omega}_{1} & \operatorname{sign}\left(\omega_{1}\right) & \omega_{1} & \omega_{1}^{2} \operatorname{sign}\left(\omega_{1}\right) & \omega_{1}^{3} & \cos \left(\theta_{1}\right) & -\sin \left(\theta_{1}\right) \\
\dot{\omega}_{2} & \operatorname{sign}\left(\omega_{2}\right) & \omega_{2} & \omega_{2}^{2} \operatorname{sign}\left(\omega_{2}\right) & \omega_{2}^{3} & \cos \left(\theta_{2}\right) & -\sin \left(\theta_{2}\right) \\
\vdots & \vdots & \vdots & \vdots & \vdots & \vdots & \vdots \\
\dot{\omega}_{n} & \operatorname{sign}\left(\omega_{n}\right) & \omega_{n} & \omega_{n}^{2} \operatorname{sign}\left(\omega_{n}\right) & \omega_{n}^{3} & \cos \left(\theta_{n}\right) & -\sin \left(\theta_{n}\right)
\end{array}\right]
$$

is the matrix containing the other measured data and

$$
\mathbf{X}=\left[\begin{array}{lllllll}
J & c_{0} & c_{1} & c_{2} & c_{3} & P_{x} & P_{y}
\end{array}\right]^{T}
$$

is the vector of the coefficients to be determined.

Then, the coefficients can be estimated as

$$
\mathbf{X}=\mathbf{M}^{+} \tau
$$

when $\mathbf{M}^{+}$denotes the Moore-Penrose pseudo-inverse of the matrix $\mathbf{M}$.

It is obvious that, in order to provide a sensible result, the trajectory used in the experiments have to span all the possible motor velocities for many times to effectively cope with measurement noise. In this paper, as a standard industrial controller is employed, the performed trajectories are point-to-point motions (with S-curve velocity profiles, as imposed by the controller) repeated many times. It has to be noted at this point that, although the polynomial model is effective to model the influence of the joint velocity on the friction torque, it does not take into account that the friction torque strongly depends also on the temperature of the joint, as it will be explained in the next section.

\section{Modelling Temperature Effect}

An important issue, that can be noted in practice during the experiments performed with an industrial manipulator (see Section 4), is that friction torque changes during robot operation. In particular, if, for example, a series of point to point motions is performed after the robot has not been used for a long time, when the friction function is determined after different time intervals, it appears that the friction term decreases, until a steadystate value is obtained for a given velocity. An illustrative example related to the second joint of the industrial robot manipulator described in Section 4 is shown in Figure 2, where the friction curves have been determined each six minutes, and it appears that after the warm-up of the robot the friction torque decreases of about $33 \%$ at high velocities. This behaviour can be associated with the temperature of the joint, which increases as the joint is more and more employed. It is therefore necessary to take into account the temperature effect in the friction model in order to compensate for the warm-up of the robot during its operations, especially if the operations start after a time interval during which the robot is at rest.

For this purpose, a modification of the polynomial model of Section 2 is proposed hereafter, by assuming that the gearbox temperature changes because the friction losses are converted into thermal power. The model is derived by starting from the thermal balance of the joint, that is,

$$
W_{a c c}=W_{i n}-W_{o u t}
$$

where $W_{a c c}$ is the accumulated thermal power in the joint, that is,

$$
W_{a c c}=C \frac{d T}{d t}
$$




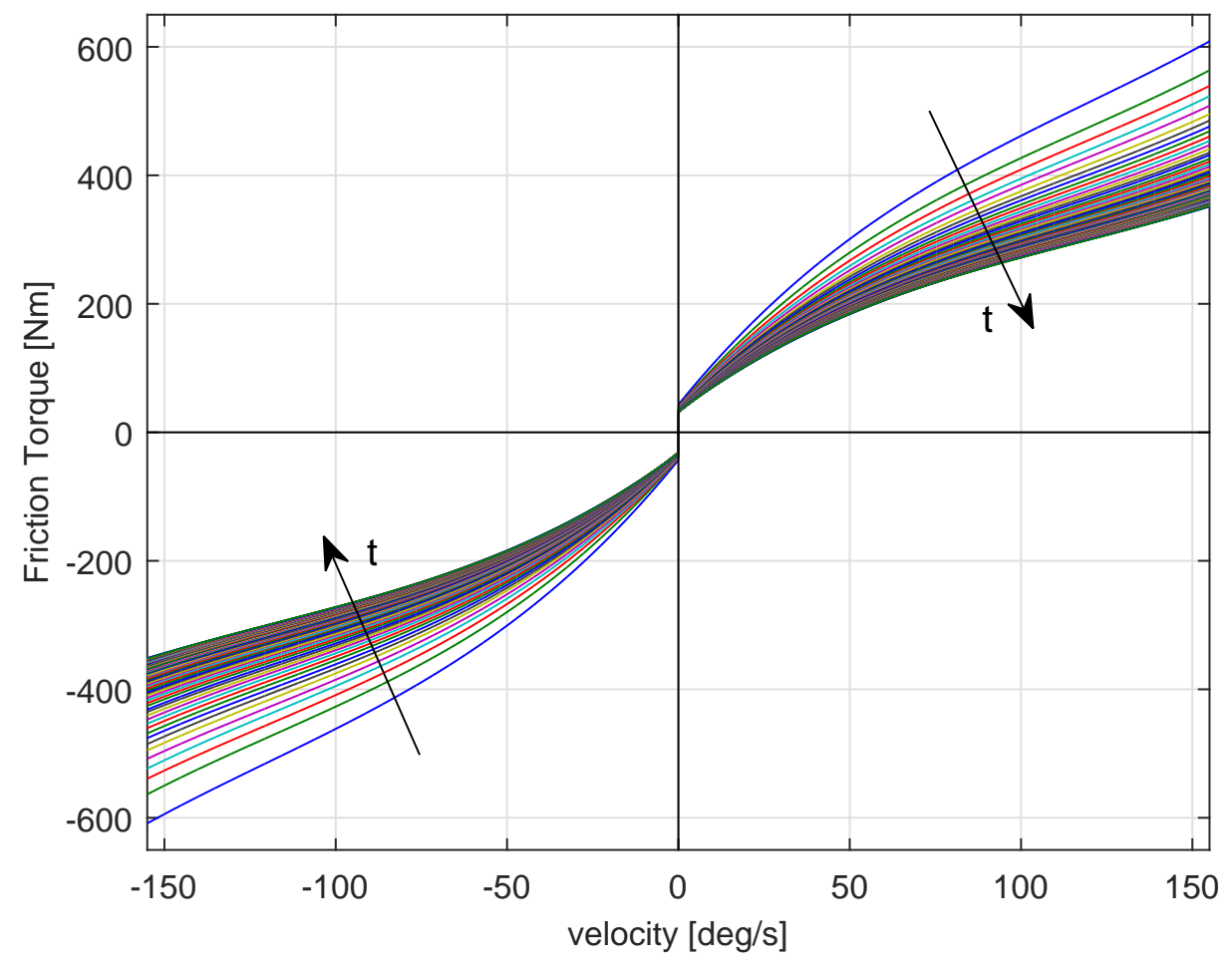

Figure 2: Modification of the friction torque curve during robot warm-up (intervals of six minutes).

where $T$ is the internal temperature of the joint and $C$ is the thermal capacity of the joint; $W_{\text {out }}$ is the dissipated thermal power

$$
W_{\text {out }}=K\left(T-T_{\text {env }}\right)
$$

where $K$ is the coefficient of thermal exchange between joint and air (we assume that this includes all the conduction and convection terms) and $T_{e n v}$ is the environmental temperature (which is assumed it can be measured and it is constant during a robot operation); $W_{i n}$ is the thermal power injected to the joint, which is assumed to be the product between the friction torque and the speed of motion, that is,

$$
W_{i n}=\tau_{f} \omega \geq 0
$$

Thus, we can write

$$
\frac{d T}{d t}=\left[\tau_{f} \omega-K\left(T-T_{e n v}\right)\right] \frac{1}{C} .
$$

Then, the proposed model is based on the assumption that friction coefficients $c_{0} \ldots c_{3}$ change linearly with temperature $T$ as

$$
c_{i}=c_{i 0}\left[\alpha\left(T-T_{0}\right)+\beta\right]
$$

where $c_{i 0}$ is the value of $c_{i}$ for $T=T_{0}$, and $\alpha$ and $\beta$ are the coefficients representing the assumed linear relation between the friction torque and the temperature. Actually, the linear dependency of the friction parameters on the temperature is not related to a rigorous physical analysis of the phenomenon, but, as it will be shown in the next sections, this simple empirical solution is effective in the description of the temperature effects from 
a practical point of view. Further, rather than representing the exact temperature in a single point, variable $T$ is an equivalent average temperature that is practically related to various different causes of friction (bearings, speed reducers, etc...) that are present in the mechanical structure. It is worth stressing that, in case of perfect modeling, it is $\beta=1$; however, a slightly different value is expected because other possible friction causes have not been considered and because of the unavoidable presence of noise in the identification phase.

If the task of the robot consists of short repetitive working cycles of duration $\Delta t$, in which the temperature can be assumed to be constant, the injected thermal power can be assumed to be constant as well, and equal to its mean value. Thus, the model assumes a linear relation between joint internal temperature and friction torque expressed as

$$
\left\{\begin{array}{c}
\frac{\Delta T}{\Delta t}=\left[\tau_{f, R M S} \bar{\omega}-K\left(T-T_{e n v}\right)\right] \frac{1}{C} \\
\tau_{f, R M S}=\tau_{f 0, R M S}\left[\alpha\left(T-T_{0}\right)+\beta\right]
\end{array}\right.
$$

where $\tau_{f, R M S}$ is the RMS value of the friction torque in a given robot operation cycle and $\tau_{f 0, R M S}$ is the RMS value of the friction torque obtained when a robot cycle is performed for the first time when the manipulator is used after it has been stopped for a long period $\left(T=T_{0} \cong T_{e n v}\right)$. Finally, $\bar{\omega}$ is the velocity that, by applying the RMS torque, would give the mean friction power and can be called 'equivalent thermal velocity'. Namely, it is the ratio between the mean friction power and the RMS friction torque, that is,

$$
\bar{\omega}=\frac{\operatorname{mean}\left(\tau_{f} \omega\right)}{\tau_{f, R M S}} .
$$

The friction torque at a given temperature is then expressed as

$$
\tau_{f}(T)=\left(c_{0}+c_{1}|\omega|+c_{2}|\omega|^{2}+c_{3}|\omega|^{3}\right) \operatorname{sgn}(\omega)\left[\alpha\left(T-T_{0}\right)+\beta\right]
$$

Thus, after the four model parameters $\alpha, \beta, K, C$ (in addition to $c_{0}, c_{1}, c_{2}, c_{3}$ ) are estimated once by performing identification experiments (see Section 5) at a given environmental temperature $T_{e n v}=T_{0}$, the model can be employed to obtain the friction torque for new robot operations (starting from an environmental temperature $\left.T_{\text {env }}\right)$.

In practice, at the beginning of a new robot operation (after the robot has been at rest), the friction torque is given by (22) with $T=T_{e n v}$. Then, at the end of the first cycle, the value of $\tau_{f 0, R M S}$ is computed and the value of $\tau_{f, R M S}$ is computed by means of (20). By also computing the value of $\bar{\omega}$ by means of (21), the change of the temperature in the joint can be calculated by means of (19).

Then, if the robot operations do not change, the value of the joint temperature can be obtained at the end of a cycle by simply applying (20) and (19) iteratively (note that $\tau_{f 0, R M S}$ is the RMS value obtained at the first iteration). The friction torque can then be estimated by applying (22) with the updated value of the joint temperature.

The differential equation (19) can be solved by considering $T(0)=T_{0}$ as initial condition. It results:

$$
T(t)=\frac{\beta \tau_{f 0, R M S} \bar{\omega}+K T_{e n v}-\alpha \tau_{f 0, R M S} T_{e n v} \bar{\omega}}{K-\alpha \tau_{f 0, R M S} \bar{\omega}}+h e^{\frac{\alpha \tau_{f 0, R M S} \bar{\omega}-K}{C} t}
$$

where

$$
h=\frac{K T_{0}-\alpha \tau_{f 0, R M S} T_{0} \bar{\omega}-\beta \tau_{f 0, R M S} \bar{\omega}-K T_{e n v}+\alpha \tau_{f 0, R M S} T_{0} \bar{\omega}}{K-\alpha \tau_{f 0, R M S} \bar{\omega}} .
$$


It appears that the time constant of the first order system is

$$
t_{c}=\frac{C}{K-\alpha \tau_{f 0, R M S} \bar{\omega}}
$$

and its knowledge can be employed to determine the maximum duration of the time interval after which the joint temperature has to be updated. This time interval has to be significantly smaller than $t_{c}$, therefore, a sensible value for it has been chosen as $t_{c} / 10$.

\section{Experimental Setup}

The robot manipulator used to perform the experiments is described in this section so that the presented results can be better evaluated. The robot is a Comau SMART NS 16 1.65 ARC (see Figure 3), and it has been designed to perform arc welding tasks. It is a serial manipulator built with an anthropomorphic kinematic structure, with six degrees of freedom and a maximum payload of 16 kilograms. It is placed on the ground for a standard working configuration.

Joints are actuated by means of six AC brushless servomotors with speed reducers. The maximum stall torque for each motor is shown in Table 1, while the type and the reduction rate of the speed reducers are shown in Table 2. The limits of the position and of the speed for each joint are represented in Table 3. Brakes are mounted directly inside the servomotors. Lubrication is made by oil for the first four axes and by grease for the last two joints.

It is worth stressing that the control architecture used for this work is the standard industrial one, called C4G, so that the proposed friction models can be implemented with a typical industrial setup. The angular position of the motors is measured by using encoders and the velocity and the acceleration of the joints are determined from the position value by using an approach based on a Savitzky-Golay filter that uses a $n$ th-order polynomial interpolation. The joint torque is determined by multiplying the measured motor current by the torque constant of the motor. The joint position controller consists of a cascade control structure where the current loop sampling period is $50[\mu \mathrm{s}]$, the velocity control loop sampling period is $200[\mu \mathrm{s}]$ and the position control loop sampling period is $400[\mu \mathrm{s}]$. The reference position values of each joint are updated at a sampling rate of $2 \mathrm{~ms}$. An accurate dynamic model of this robot is available [33,34].

\begin{tabular}{|c|c|c|c|}
\hline Axis & Stall Torque [Nm] & Voltage [V] & Power [kW] \\
\hline 1 & 3.3 & 325 & 1.41 \\
\hline 2 & 9 & 325 & 2.50 \\
\hline 3 & 3.3 & 325 & 1.41 \\
\hline 4 & 0.75 & 325 & 0.33 \\
\hline 5 & 0.75 & 325 & 0.33 \\
\hline 6 & 0.75 & 325 & 0.33 \\
\hline
\end{tabular}

Table 1: Characteristics of the motors. 


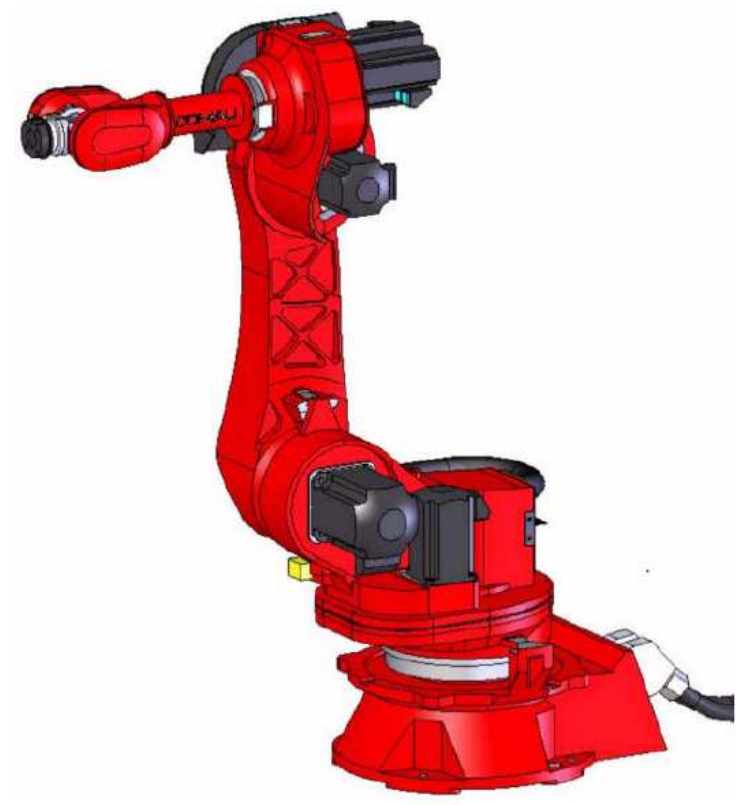

Figure 3: The robot used in the experiments.

\begin{tabular}{|c|c|c|}
\hline Axis & Reduction rate & Type \\
\hline 1 & -181.5873 & Gears \\
\hline 2 & 159.2609 & Cycloidal \\
\hline 3 & 169.4737 & Cycloidal \\
\hline 4 & 72.5860 & Gears \\
\hline 5 & 78.7500 & Harmonic Drive \\
\hline 6 & -50.0000 & Harmonic Drive \\
\hline
\end{tabular}

Table 2: Reduction rates of the speed reducers for each joint.

\begin{tabular}{|c|c|c|}
\hline Joint & Position limits [deg] & Speed limits [deg/s] \\
\hline 1 & \pm 180 & 155 \\
\hline 2 & $+155 /-60$ & 155 \\
\hline 3 & $+110 /-170$ & 170 \\
\hline 4 & \pm 2700 & 360 \\
\hline 5 & \pm 120 & 350 \\
\hline 6 & \pm 2700 & 550 \\
\hline
\end{tabular}

Table 3: Position and speed joints limits.

\section{Identification}

As already mentioned, the polynomial friction function parameters (see (2)) can be estimated once, at the known environmental temperature, by applying the simple least squares procedure described in Section 2 . In 


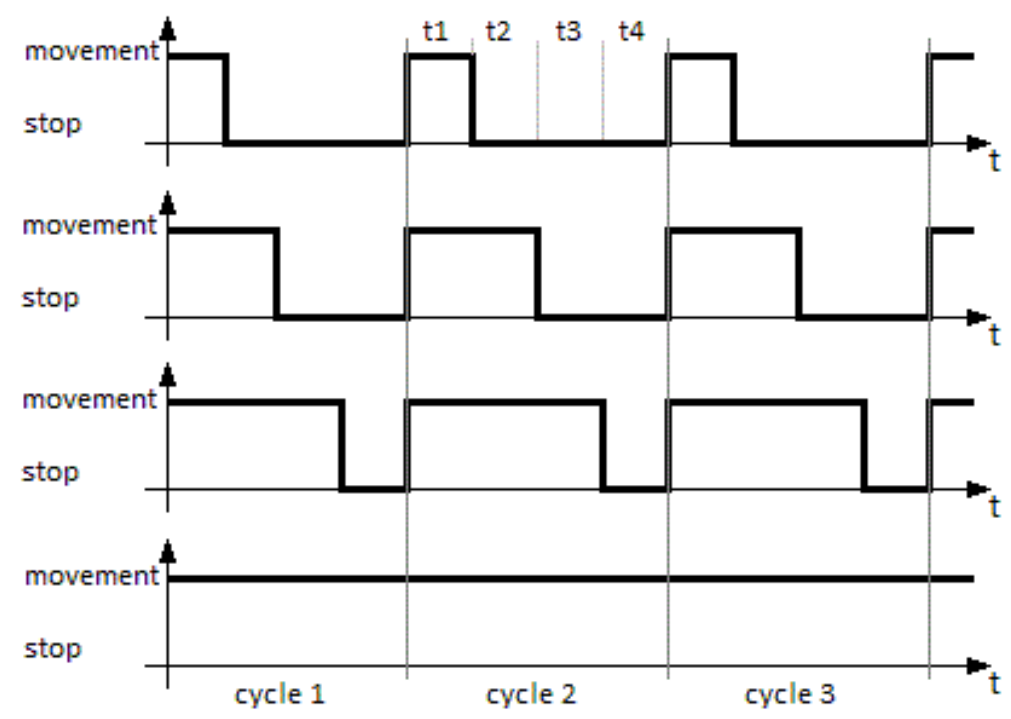

Figure 4: The different cycles robot operations for the identification of the model $\left(W_{\text {in }}=25 \%, 50 \%, 75 \%\right.$, $100 \%)$.

this case the simple experiments have to be performed when the robot has been at rest for a long time so that the temperature of the joint is virtually equal to the temperature of the environment.

Then, in order to estimate the parameter of the model (19)-(20) the robot has to be moved by spanning the widest possible range of velocities and injected thermal power and by determining the corresponding friction functions. A simple practical solution for this purpose has been developed by applying for each joint four different kinds of robot operations, where for each operation the robot starts after a long period (one night) of inactivity $\left(T=T_{0} \cong T_{\text {env }}\right)$. In particular, cycle times of four minutes have been considered and each one has been divided in subintervals of one minute. The four cases differ because the same movements of the robot are performed in each subinterval, but the number of subintervals in which the robot is moved in each cycle is different, as shown in Figure 4. In other words, it can be said that each case represents a robot operation with a different duty cycle. In this way, the thermal power injected into the joints is different; for a given joint temperature, the thermal power is proportional to the duty cycle. In each subinterval of the cycle time, point to point motions from $-45 \mathrm{deg}$ to $70 \mathrm{deg}$ and viceversa are performed, in the case of the first two joints, with six different peak velocities, namely, $5 \%, 24 \%, 43 \%, 62 \%, 81 \%$, and $100 \%$ of the maximum velocity allowed the motor. The estimated temperature is updated at the end of each cycle, that is, each four minutes.

It is worth stressing that the motion of the joints can be performed separately or at the same time. In the first case the friction torque is calculated by means of the method described in Section 2, while in the latter case the dynamic model (1) of the robot has to be employed in order to determine the friction torque for each joint.

The parameters of the model (19)-(20) are estimated by considering all the experiments and by minimizing the sum of the square errors, that is the difference between the friction force estimated by the experimental data and the one estimated by the model for all the different velocities. For this purpose, the Matlab function fmincon has been used. Results related to the second joint of the robot are considered in this section as this 
joint is the most relevant for the gravitational force; the other joints have in any case a similar behaviour. The obtained values of the parameters are $\alpha=-0.00937, \beta=0.91708, K=2.75614$ [W/K], $C=18086.82$ $[\mathrm{J} / \mathrm{K}]$. The obtained results are shown in Figure 5 , where the four different duty cycles are considered and the measured and the estimated friction torques are compared at different time intervals (in particular after 4 and 304 minutes). The temperature evolution determined for the four different duty cycles are plotted in Figure 6 where it can be seen that, as expected, the temperature increment is the more relevant the longer are the time intervals in which the robot is in motion. 

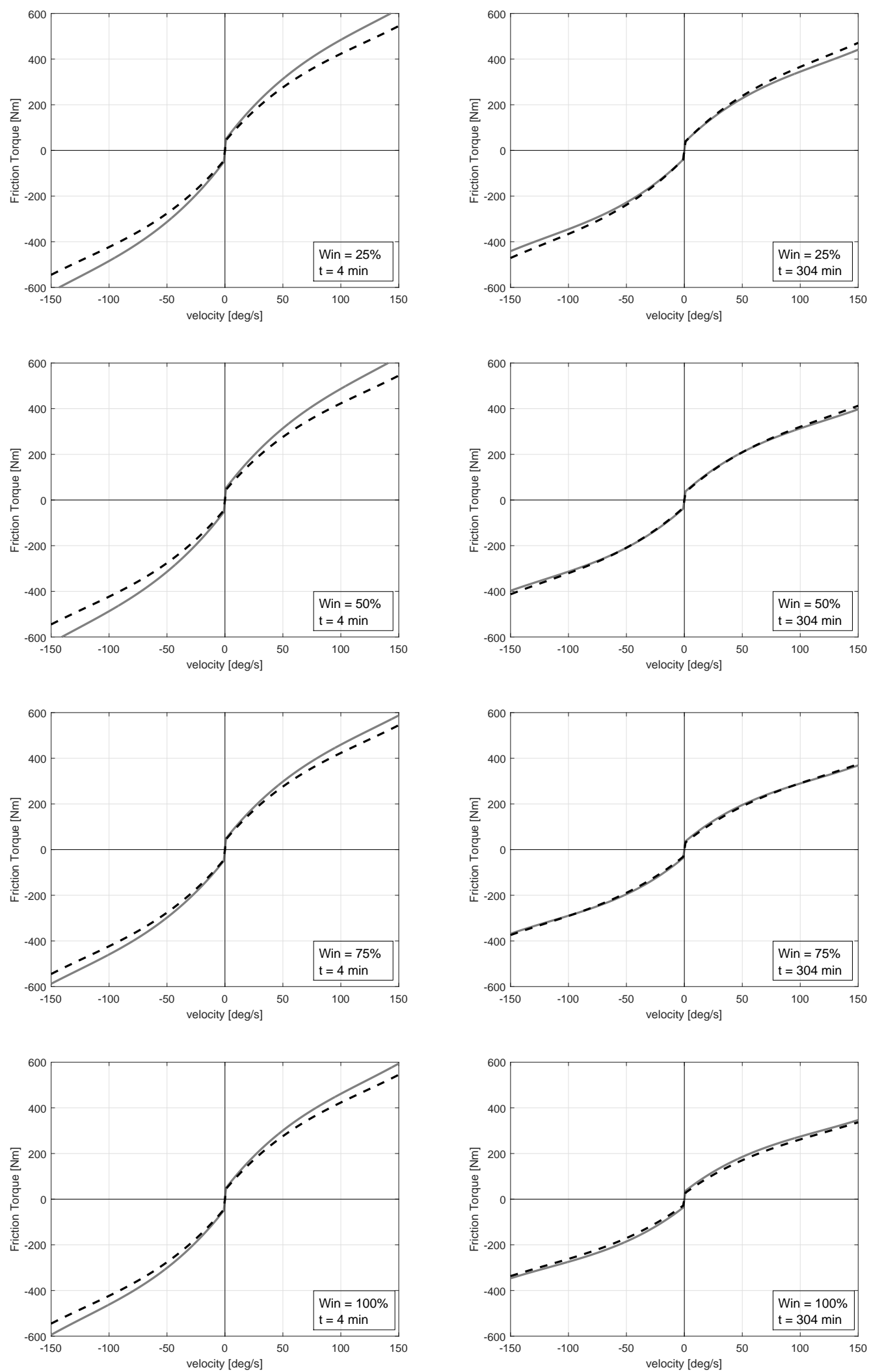

Figure 5: Identification results. Friction torque obtained from experimental data (grey solid line) and identified one (black dashed line) for joint 2 with different injected thermal power and after different time intervals. 


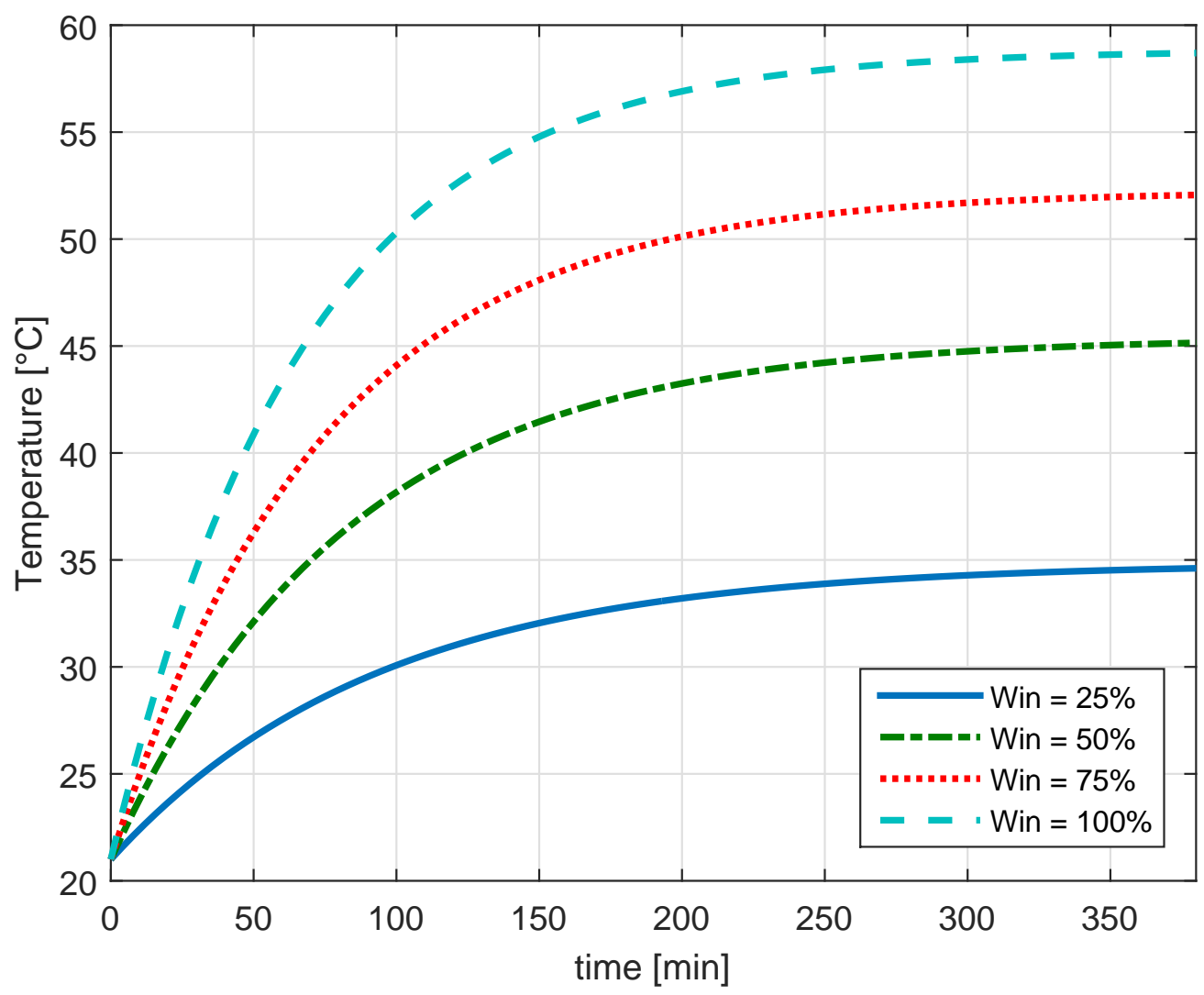

Figure 6: Estimated joint temperature for different robot operations, that is, for different input thermal powers injected into the joint.

Note that the joint internal temperature, even if it is obviously greater than the environmental one, it is in the same order of magnitude (note that in any case manipulators have, in general, a thermal protection in case of overheating, which means that the robot stops if the temperature exceeds a given threshold). In such a range the lubricant viscosity can be considered as linearly dependent on the temperature with a good approximation [25]. Furthermore, although by using (20) there is the theoretical possibility that the viscosity becomes zero or even negative for high temperature, in practice this never happens. The determined values of the time constants are $t_{c}=101.38 \mathrm{~min}$ for robot cycles where the robot is moving for one minute and it is stopped for three minutes (that is, $W_{i n}=25 \%$ ), $t_{c}=89.51 \mathrm{~min}$ for $W_{i n}=50 \%, t_{c}=81.58 \mathrm{~min}$ for $W_{i n}=75 \%$, and $t_{c}=73.96$ min for $W_{i n}=100 \%$, when the robot is always in motion (note that, as shown in (25), the time constant $t_{c}$ depends on the injected thermal power $\left.W_{i n}=\tau_{f 0, R M S} \bar{\omega}\right)$. It is in any case evident that updating the value of the temperature each 4 minutes is sufficient, taking into account the dynamics of the system.

From the identification results it appears that the proposed model is capable of describing very well the temperature effect on the friction torque. Indeed, the friction torque decreases from the beginning of the operations after a period of rest, until a given time when the temperature function becomes almost constant and therefore the friction does not change significantly anymore. The obtained model has then been validated by applying to the second joint point-to-point motions from -45 to $+70 \mathrm{deg}$, starting virtually from the same temperature $T_{0} \cong T_{\text {env }}$ of the identification experiments. These have been repeated for many cycles of three minutes (by 

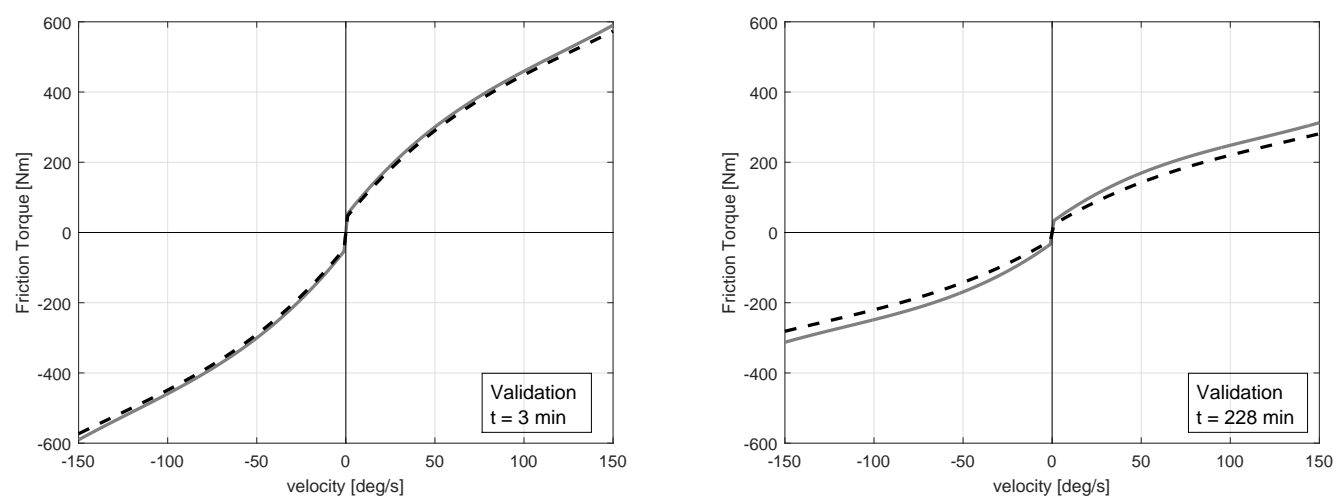

Figure 7: Validation results. Friction torque obtained from experimental data (grey solid line) and identified one (black dashed line) after different time intervals.

\begin{tabular}{|c|c|c|c|c|}
\hline Time & With Temperature & Without Temperature & With Temperature & Without Temperature \\
\hline$[\mathbf{m i n}]$ & $\mathbf{s t d}(\mathbf{e r r})[\mathbf{N m}]$ & $\mathbf{s t d}(\mathbf{e r r})[\mathbf{N m}]$ & $\operatorname{var}(\mathbf{e r r}) / \mathbf{v a r}\left(\boldsymbol{\tau}_{f}\right)[\%]$ & $\operatorname{var}(\mathbf{e r r}) / \mathbf{v a r}\left(\boldsymbol{\tau}_{\boldsymbol{f}}\right)[\%]$ \\
\hline 3 & 48.4991 & 49.4382 & 2.59 & 2.69 \\
\hline 33 & 43.6194 & 106.0872 & 4.00 & 23.69 \\
\hline 63 & 45.5116 & 128.5030 & 5.40 & 43.06 \\
\hline 93 & 50.1176 & 140.2410 & 7.30 & 57.18 \\
\hline 123 & 53.3136 & 147.8523 & 8.88 & 68.28 \\
\hline 153 & 55.3255 & 152.3702 & 9.97 & 75.63 \\
\hline 183 & 57.0318 & 154.7700 & 10.79 & 79.49 \\
\hline 213 & 58.2405 & 156.4537 & 11.39 & 82.17 \\
\hline 243 & 58.7912 & 157.6204 & 11.71 & 84.18 \\
\hline 273 & 59.3544 & 158.1072 & 11.96 & 84.46 \\
\hline
\end{tabular}

Table 4: Standard deviation of the torque error and percentage of unexplained variance for joint 2, both computed on the validation trials with and without taking into account the temperature change. Data are computed every 30 minutes of robot operations without filtering.

never stopping the robot) with velocities equal to $10 \%, 25 \%, 40 \%, 55 \%, 70 \%, 85 \%$ and $100 \%$ of the maximum velocity in each cycle. The results obtained by calculating the friction torque with the method of Section 2 are then compared with those obtained by applying the four parameters model estimated in the previous section. Results are shown in Figure 7, where the plots are related to the comparison after 3 and 228 minutes.

In Table 4 it is possible to see the standard deviation of the estimated friction torque error computed by considering the joint temperature change and without considering it for joint 2 . The fraction of unexplained variance it is also be shown. It appears that the standard deviation of the model error is significantly smaller when the temperature is considered; furthermore, the percentage of the physical data not explained by the model is smaller when the temperature is taken into account. These results confirm again the effectiveness of the proposed model and of the adopted identification procedure. 


\section{Temperature Observer}

As already mentioned, the environmental temperature is assumed to be measured and the parameters $\alpha, \beta, K$, $C$ (in addition to $c_{0}, c_{1}, c_{2}, c_{3}$ ) of the model can be estimated just once for a robot manipulator by means of the identification procedure described in Section 5 (actually, the procedure should be performed again if the physical properties of the lubricant change significantly over the time). Thus, the determination of the current joint temperature (and therefore the estimation of the friction torque) depends on the use of the robot and on the initial joint temperature. The latter is in general difficult to estimate (only if the robot has been at rest for long time it can be assumed that the joint temperature is equal to the environmental temperature) and as already mentioned, it is rather impractical to measure it directly. For this reason, a temperature observer is necessary to compensate for a wrong estimation of the initial condition [21]. Further, the computation of $\bar{\omega}$ in (19) requires the knowledge of $\tau_{f}$ and therefore the temperature has to be computed in an accurate way.

The temperature observer uses, as innovation, the difference between the friction torque $\tau_{f}$ and its estimation $\hat{\tau}_{f}$ computed with current temperature estimation. It is important to note at this point that, in general, the measured torque $\tau$ is the sum between the friction term $\tau_{f}$ and the term $\tau_{d}=\mathbf{F}(\boldsymbol{\theta}, \dot{\boldsymbol{\theta}}, \ddot{\boldsymbol{\theta}})$ composed by the inertial, Coriolis and gravitational torques, which can be estimated from experimental tests [16,34]. Thus, the friction torque can be computed as $\tau_{\boldsymbol{f}}=\boldsymbol{\tau}-\boldsymbol{\tau}_{\boldsymbol{d}}$. The temperature estimation $\hat{T}$ is suitably updated at each sampling time as

$$
\hat{T}(i+1)=\hat{T}(i)+\left[\left[\tau_{f, R M S}(i) \bar{\omega}(i)-K\left(\hat{T}(i)-T_{e n v}\right)\right] \frac{1}{C}-\eta\left(\tau_{f, R M S}(i)-\hat{\tau}_{f, R M S}(i)\right)\right] t_{s}
$$

where $i$ is the current sampling time instant, $\tau_{f, R M S}$ is the RMS friction torque value determined by considering $N$ previous samples of the measured friction torque, $\hat{\tau}_{f, R M S}$ is the RMS value of the previous $N$ samples of the estimated friction torque (obtained by applying (22)), $\bar{\omega}$ is the velocity computed using (21) for $N$ previous samples and $t_{s}$ is the sampling period. The proposed scheme is shown in Figure 8.

The parameters to set in order to apply the observer technique are the number $N$ of previous samples to use for the computation of the RMS torque values and the observer gain $\eta$. The former can be fixed in order to consider a reasonably short time interval that gives at the same time a consistent result (note that the higher is

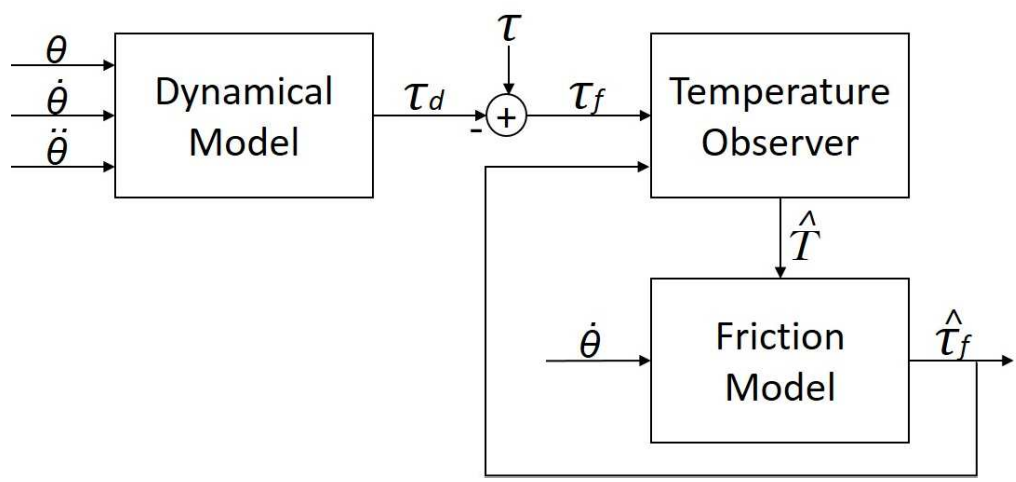

Figure 8: Scheme of the temperature observer used for friction estimation. 
the value of $N$ the higher is the filtering effect). The latter can be selected by making the considerations typical of observer schemes in the presence of noise, that is, a small value provides a small bandwidth of the observer that implies a smoother but slower convergence to the correct temperature value. On the contrary a high value provides a large bandwidth that implies a faster but also more oscillatory convergence (and a too high value might lead the system to instability). In any case, it is worth stressing that the observed temperature allows the prediction of the friction torque for new values of velocities that have not been previously used during the robot operations, as it will be shown in the next section. In fact, the interpolation capabilities of the estimated polynomial friction function can be exploited only with a correct estimation of the temperature (see Figure 2).

\section{Experimental Results}

\subsection{Evaluation of the model}

Two kinds of experiments have been performed in order to verify the effectiveness of the proposed model:

- a sequence of single joint repetitive point to point motions, performed in three different phases. For joint 2 , in the first phase, of duration 4960 [s], the peak speed is fixed at $40 \%$ of the maximum joint speed. Then, the peak speed is suddenly changed to $80 \%$ of the maximum joint speed and this is kept for 2790 [s]. Finally, the peak speed is changed again suddenly to $20 \%$ of the maximum one for other 4805 [s] (see Figure 9). In the case of joint 1 times are different due to the fact that the maximum range of motion is bigger. In that case time intervals are 7632 [s] for the motion at $40 \%$ of the maximum velocity, 4116 [s] for the motion at $80 \%$ of the maximum velocity and 7089 [s] for the last motion, that is for the one at $20 \%$ of the maximum joint velocity;

- a repetitive motion that simulates a pick and place task in which the robot end effector is moved by following 6 different linear trajectories, three of them from left to right and three of them from right to left, linking repetitively the following task space coordinates (the first three coordinates are the frame position w.r.t. the base frame [mm], and the second ones are the ZYZ Euler angles [degree]): (1000, $-600,600,10,180,60),(1000,-600,1200,10,180,60),(1000,600,1200,10,180,60)$ and $(1000,600$, $600,10,180,60)$. The end effector peak velocity during the hoisting and lowering phases are $30 \%$ of the maximum end-effector, while the peak velocity during the lateral movement is the maximum allowable end-effector velocity.

It is worth stressing that for single joint motions the complete dynamic model of the robot is not relevant while its knowledge is essential for the pick and place motion. Results related to the first and to the second joint of the robot are shown for both tasks. Note that data have not been filtered in any way. Starting from the single joint motion results, the case where the robot starts working after a long rest period is first considered. In this way the initial joint temperature is virtually the same of the environmental temperature. The case where the speed changes after the first and second time intervals are highlighted in Figure 10. As it is possible to observe, the estimated friction torque is quite similar to the measured one along the time and also after the speed changes. This means that the friction model is capable of describing well the dependence of friction torque on the temperature that increases during the robot operations. The influence of the temperature is evident by also 


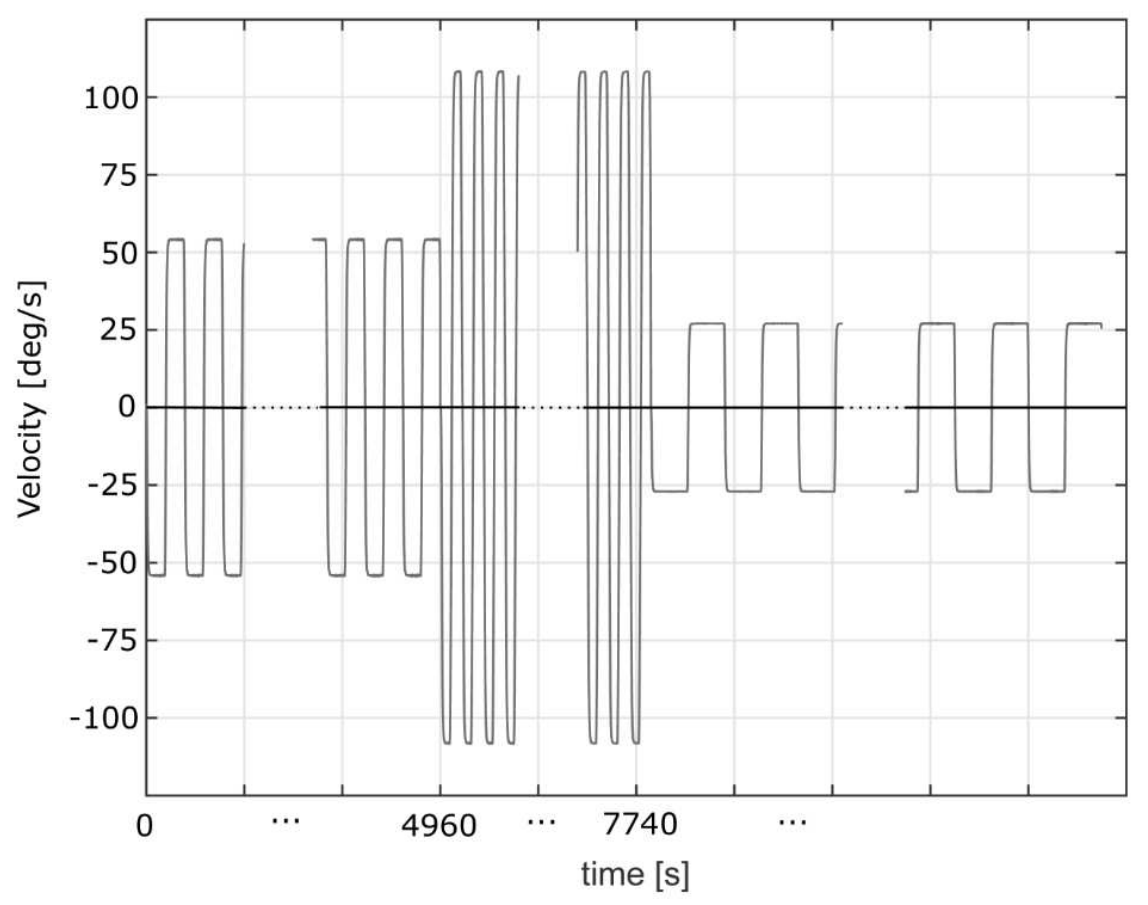

Figure 9: The considered single joint repetitive motion used in experimental campaign.

comparing the left and right parts of Figure 10 or, alternatively, the left and right parts of Figure 11. In fact, in these cases the speed of motion is fixed and after a lot of cycles during which temperature has changed, the model fits well the real data. This fact is clearly understandable by taking into account the friction torque at the end of the left part of Figure 11, which is about $300 \mathrm{Nm}$, and the one at the beginning of the right part of Figure 11, which is about $240 \mathrm{Nm}$.
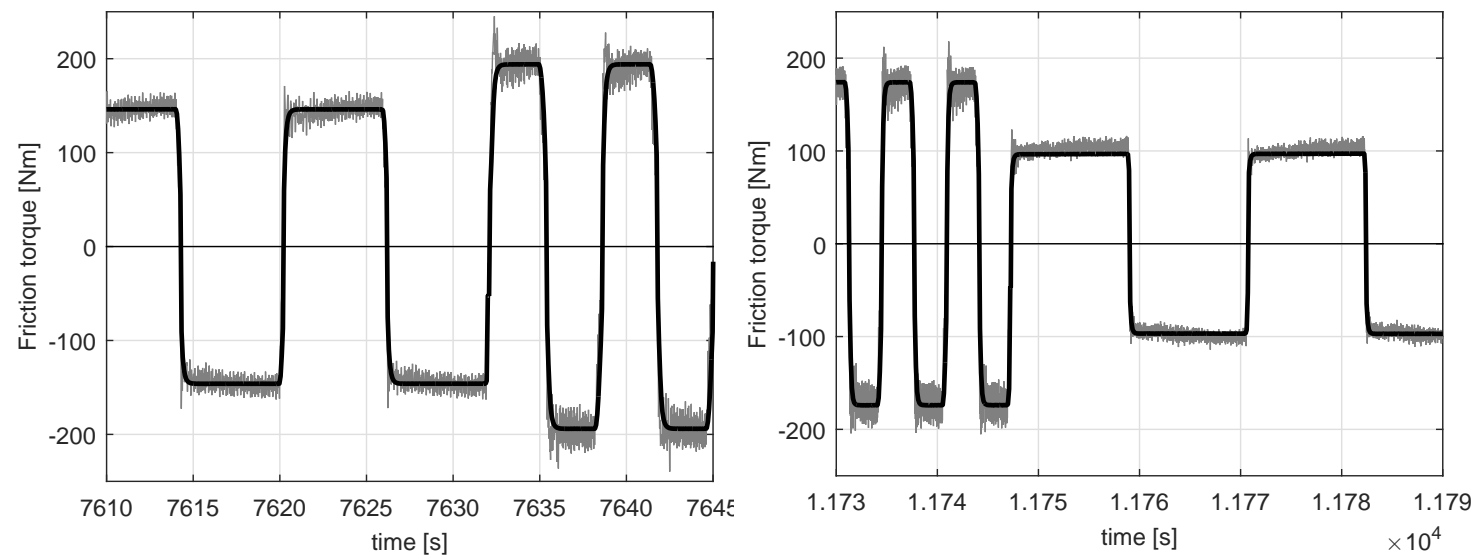

Figure 10: Friction torque obtained from experimental data (grey thin line) and estimated one (black thick line) for joint 1 during the first speed change (single joint motion experiment). 

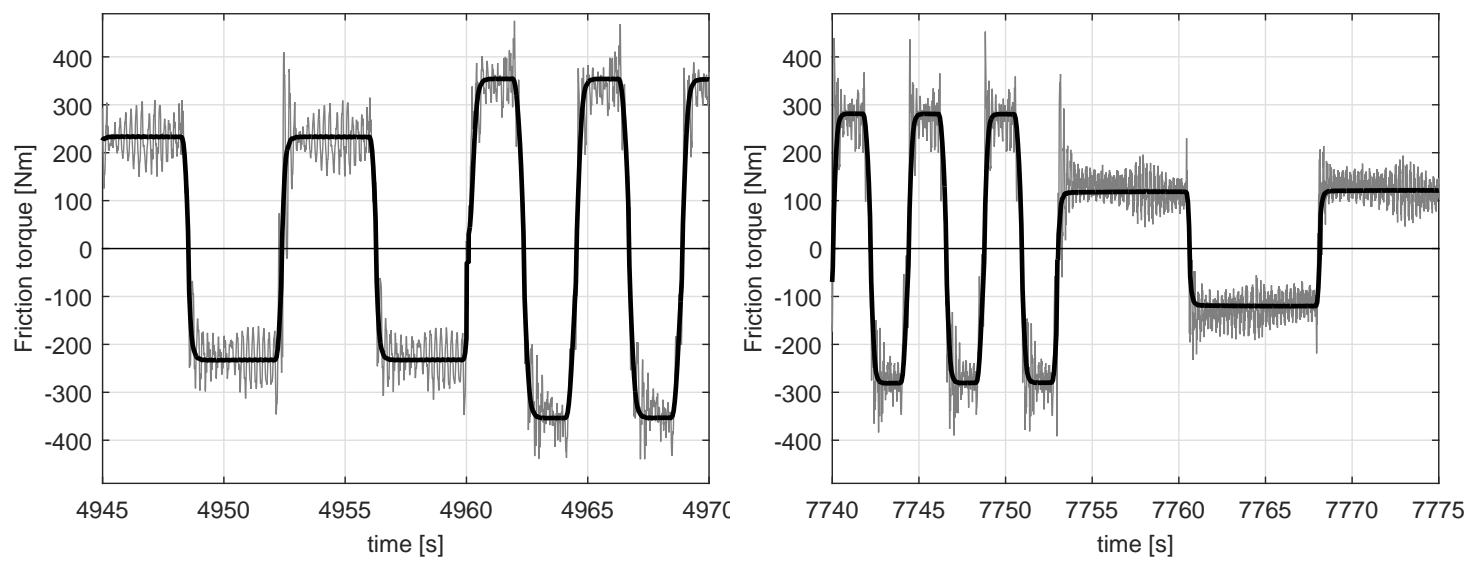

Figure 11: Friction torque obtained from experimental data (grey thin line) and estimated one (black thick line) for joint 2 during the first speed change (single joint motion experiment).

\subsection{Evaluation of the observer-based strategy}

The motion of joint 2 has been also employed to verify the effectiveness of the observer based strategy. In particular, a series of experiments has been performed where the motion starts after the robot has worked for 30 minutes so that the joint temperature is higher then the environmental one. As the latter is assumed as initial condition, this results in an error that has to be compensated. The measured and estimated friction torques for $N=20$ and for different values of $\eta$ (that is, $5 \cdot 10^{-3}, 5 \cdot 10^{-2}$ and $5 \cdot 10^{-1}$ ) are shown in Figure 12 together with the corresponding estimated joint temperature. It appears that the estimated temperature converges to the correct value after an initial transient and as expected, decreasing the value of $\eta$ yields an increment of the convergence velocity but also an increment of oscillations as the system can be too aggressive and therefore the effect of noise and disturbances could be detrimental. In order to evaluate the effect of the variation of the design parameter $N$, other experimental results are shown in Figure 13 with the same values of $\eta$ as before but with $N=40$. It can be observed that, as expected, decreasing the value of $N$ yields an increment of the sensitivity to measurement noise and disturbances. In any case, it turns out that both the design parameters have predictable effects and can be therefore fixed quite simply for a given robot, in order to obtain a convergence time of just a few tenths of seconds.

Regarding the results obtained by considering the pick and place repetitive motion, the estimated and measured friction torques for the joints 1 and 2 are shown in Figure 14 and 15 respectively for different time intervals (after 3 and after 35 minutes from the beginning of the task). Also in this case it is possible to observe that the proposed model is effective in estimating the role of the temperature on the friction term.

It is worth noting that in Figure 14 in certain time intervals the estimated friction torque is completely different from the measured one. This happens because in these intervals the motor velocity is zero and, due to the static friction phenomenon, the friction torque can assume any value between the static friction coefficients $-c_{0}$ and $+c_{0}$. Thus, it has been chosen to show an estimated friction torque equal to its extremum. 

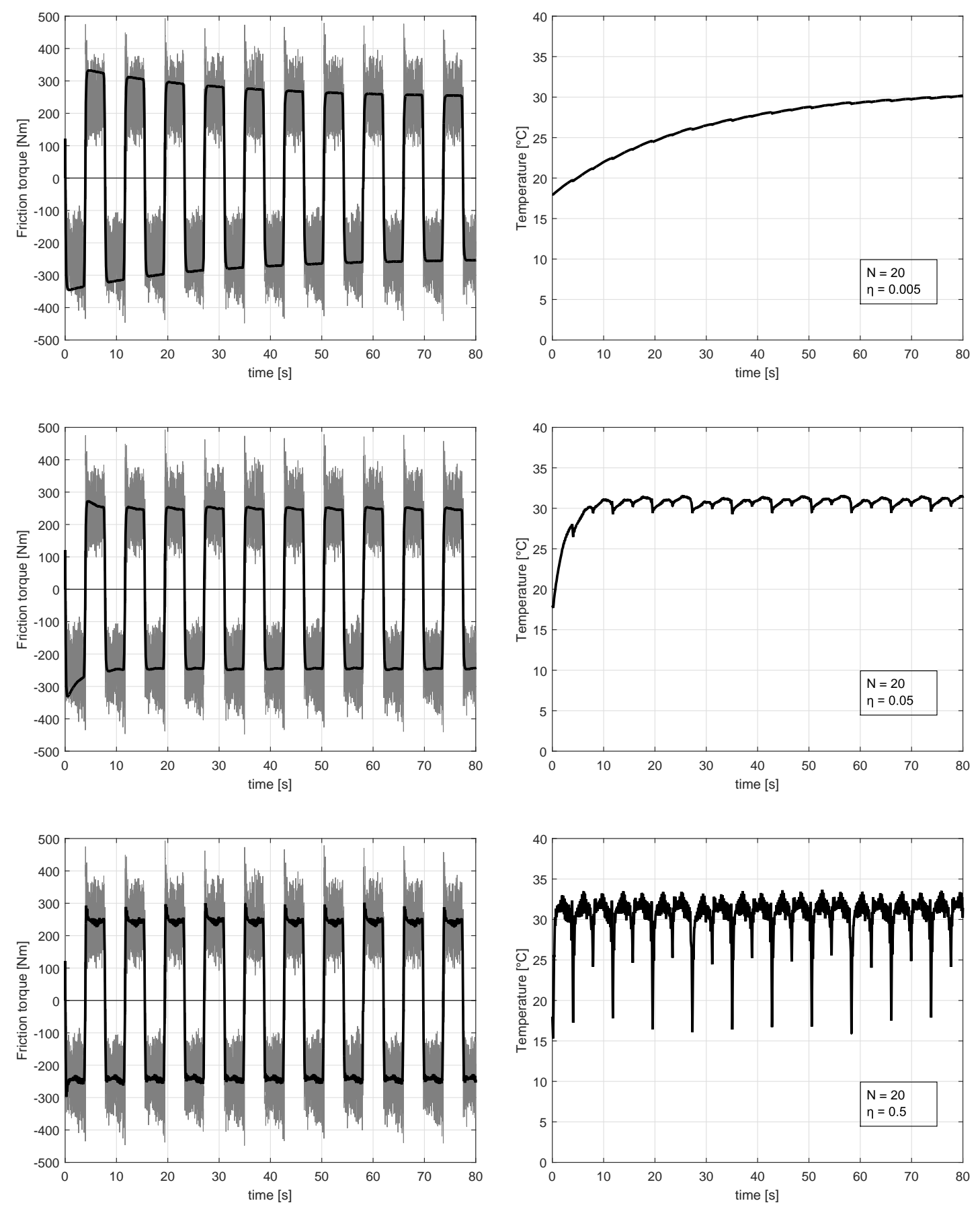

Figure 12: Friction torque obtained from experimental data (grey thin line) and estimated one (black thick line) for joint 2, and related estimated joint internal temperature (blue line), starting from a wrong initial condition with $\eta=5 \cdot 10^{-3}$ (top), $\eta=5 \cdot 10^{-2}$ (middle), $\eta=5 \cdot 10^{-1}$ (bottom) and $N=20$ (single joint motion experiment). 

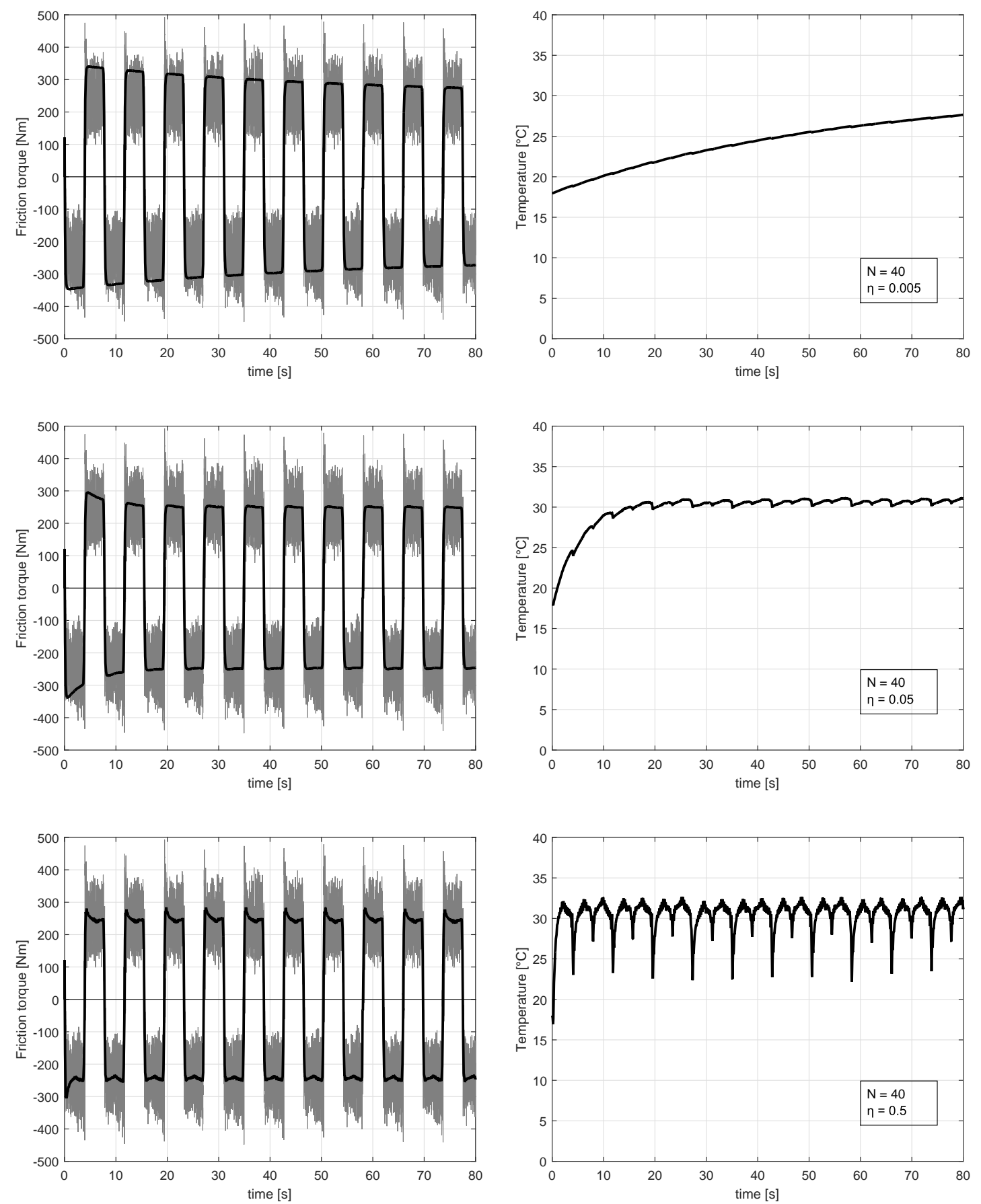

Figure 13: Friction torque obtained from experimental data (grey thin line) and estimated one (black thick line) for joint 2, and related estimated joint internal temperature (blue line), starting from a wrong initial condition with $\eta=5 \cdot 10^{-3}$ (top), $\eta=5 \cdot 10^{-2}$ (middle), $\eta=5 \cdot 10^{-1}$ (bottom) and $N=40$ (single joint motion experiment). 

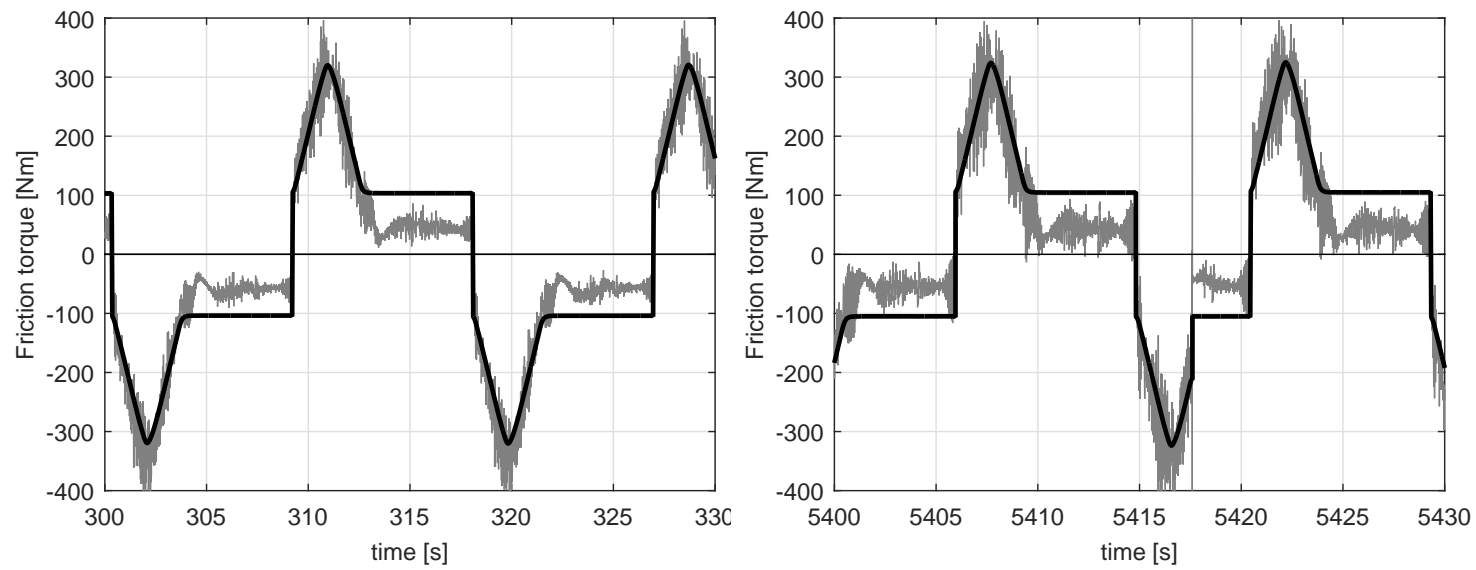

Figure 14: Friction torque obtained from experimental data (grey thin line) and estimated one (black thick line) for joint 1 during the pick and place motion.
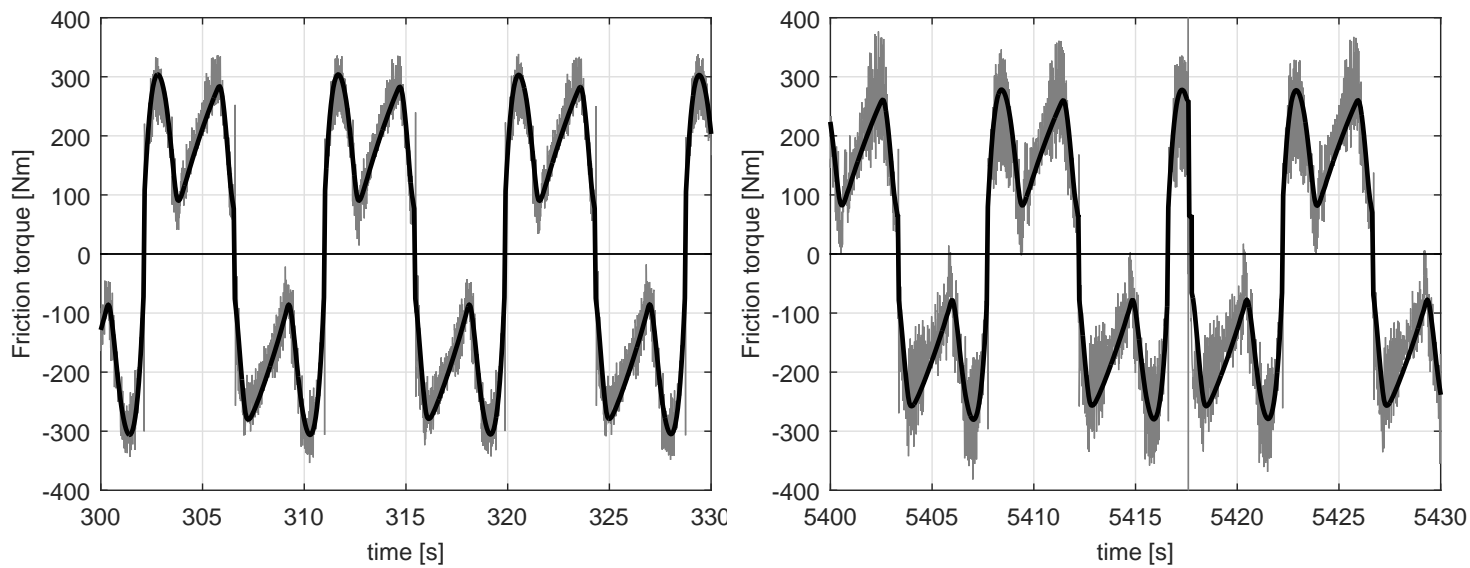

Figure 15: Friction torque obtained from experimental data (grey thin line) and estimated one (black thick line) for joint 2 during the pick and place motion. 


\subsection{Discussion}

Regarding the proposed modeling and identification technique, it has to be stressed that the observer is used only for the estimation of the (initial) joint temperature and not for the complete friction model.

In any case, the use of an on line friction estimation technique (for example by means of a recursive leastsquares procedure or by means of a Kalman filter) has been recognized to be critical in industrial robotics because, in general, the result strongly depends on an accurate dynamic model of the manipulator and the presence of external forces (disturbances) might impair the accuracy of the estimation.

Regarding the first issue, the development of advanced techniques has made accurate robot dynamic models available $[16,34]$ so that they can be safely exploited for the considered problem, as shown in the previous subsection. Regarding the second issue, it is necessary to discuss better the role of the observer, which is essential in order to overcome the lack of an additional temperature sensor. For this purpose, the following experiment has been performed. The first joint of the robot has been cyclically moved between 0 and 120 degrees with different peak velocities. The observer has been applied only for the first 120 seconds (the time interval that is necessary to estimate the intial joint temperature) and then has been disconnected.

The results plotted in Figure 16 show that, even after two hours of motion without applying the observer and just updating the temperature value according to equations (19)-(20) the estimation of the friction torque is still satisfactory (demonstrating the effectiveness of the model) while some decrement of the performance can be detected after four hours. The relevance of the results can be understood better by comparing the previous experiment with a similar one where, however, the temperature is not updated and the initially estimated friction function is always considered in the overall time interval of the experiment (see Figure 17). It appears that it is worth using the model that includes the temperature effect also in the cases when the observer cannot be used, for example when external forces have been applied (for example in pick-and-place operations).

In any case, in order to avoid the decrement of performance that is unavoidable if the devised friction model is applied in an open loop fashion, the observer can be inserted seldom for a short time interval when a free motion without any additional load occurs. This solution has been applied in the experiment of Figure 18, in which the same task of Figure 16 has been applied but the observer is connected for 120 seconds after 2 hours and for other 120 seconds after 4 hours. It appears that a short time interval is sufficient to estimate again the correct (lower) joint temperature and to recover the performance.

Finally, another interesting experiment has been performed by always using the observer in all the time interval but a constant torque disturbance (which can be considered as the result of a force disturbance acting on the end-effector) of $50 \mathrm{Nm}$, that is a value around $30 \%$ of the friction torque, has been added to joint 1 of the robot for 120 seconds after two and for hours of robot operations. Results plotted in Figure 19 show that the presence of a constant torque disturbance causes, as expected, an underestimation of the temperature, which yields a decrement in the accuracy of the estimation of the friction term, namely, an estimation bias. However, the estimation error is quickly reduced as soon as the external force stops its action.

From all the presented experiments, it can be deduced that the model is effective in describing the role of the temperature in the friction variation and that the temperature sensor in the joint can be substituted with the observer, provided an appropriate strategy is employed in order to connect the observer when the task does not present external forces. Indeed, the time needed by the observer to estimate the joint temperature is much 

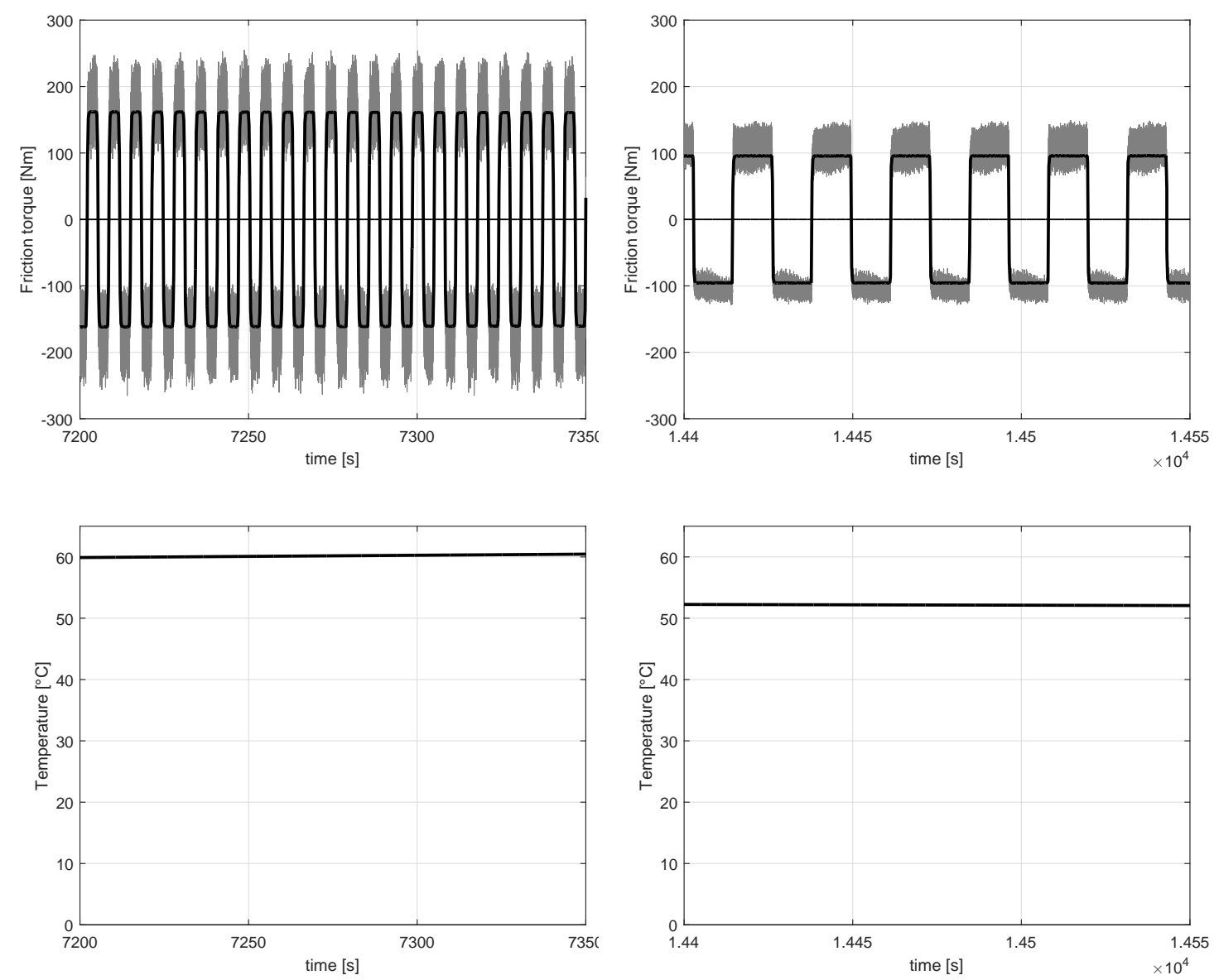

Figure 16: Friction torque obtained from experimental data (grey thin line) and estimated one (black thick line) for joint 1 without the application of the observer after 2 (left) and 4 (right) hours, and related temperature.

smaller than the time interval when the model can be applied without the observer. This makes the application of such a strategy fairly simple. For example, if a task constantly involves the presence of external forces, such as a pick-and-place task, it is possible to turn on the observer every few hours adding, if not already present, a short (less than a minute) free motion to re-estimate the joint temperature. 

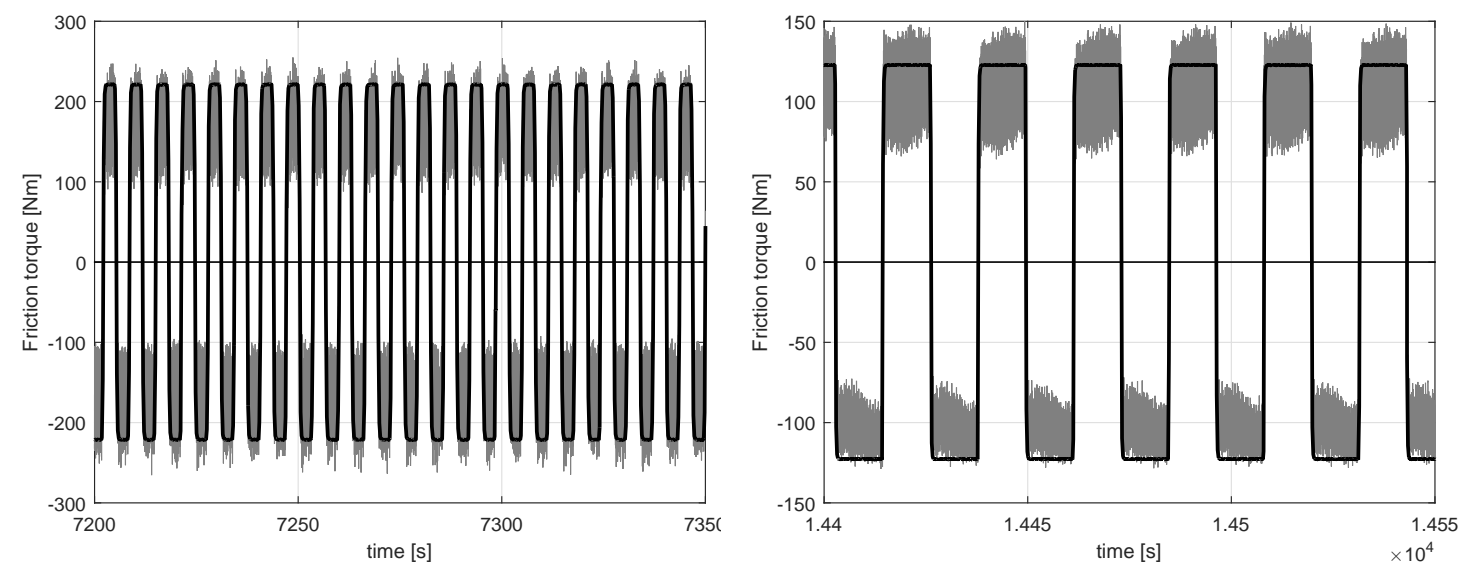

Figure 17: Friction torque obtained from experimental data (grey thin line) and estimated one (black thick line) for joint 1 without taking into account the temperature variation after 2 (left) and 4 (right) hours respectively.
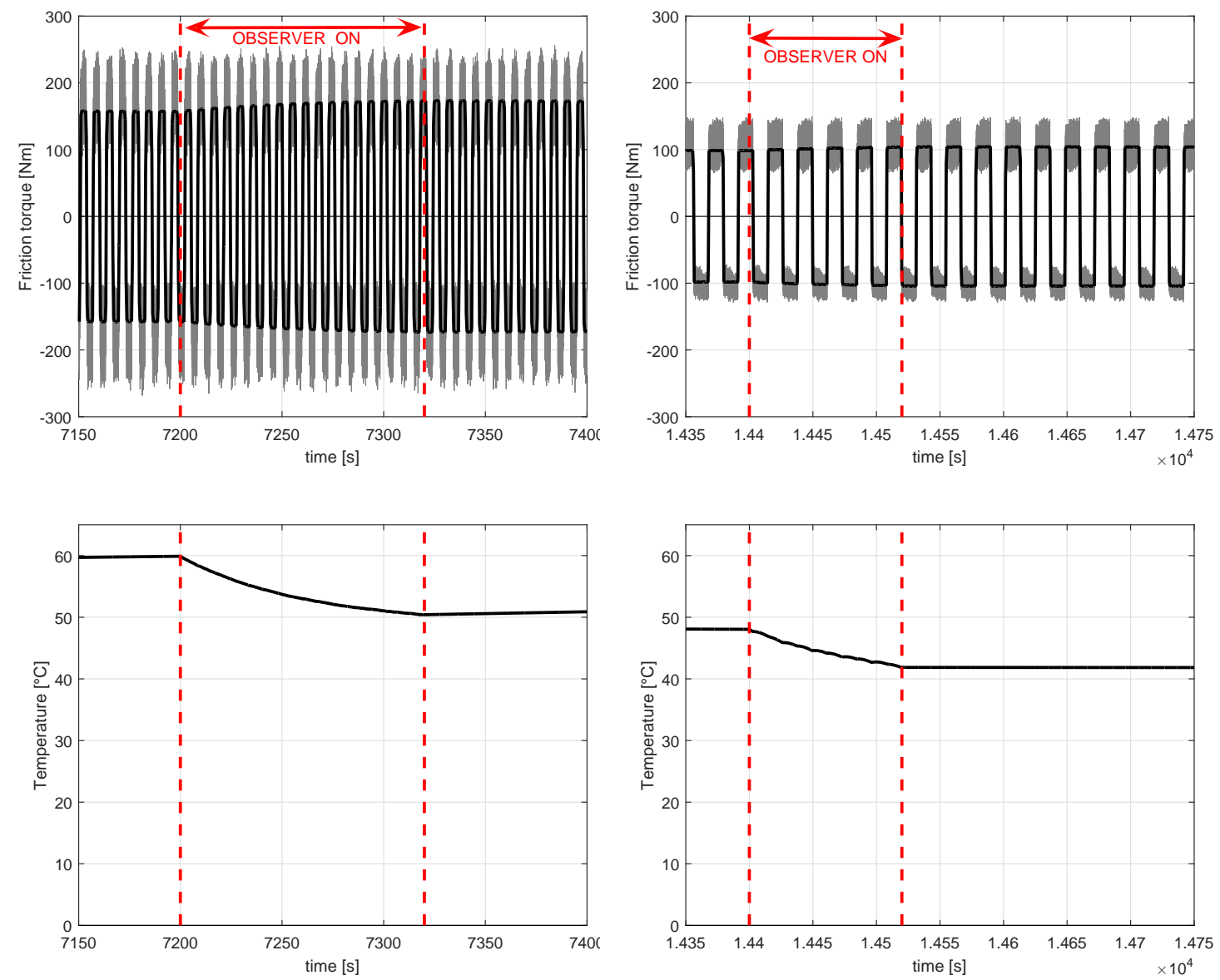

Figure 18: Friction torque obtained from experimental data (grey thin line) and estimated one (black thick line) for joint 1 during the second and third application of the observer for 120 seconds after 2 (left) and 4 (right) hours respectively, and related temperature. 

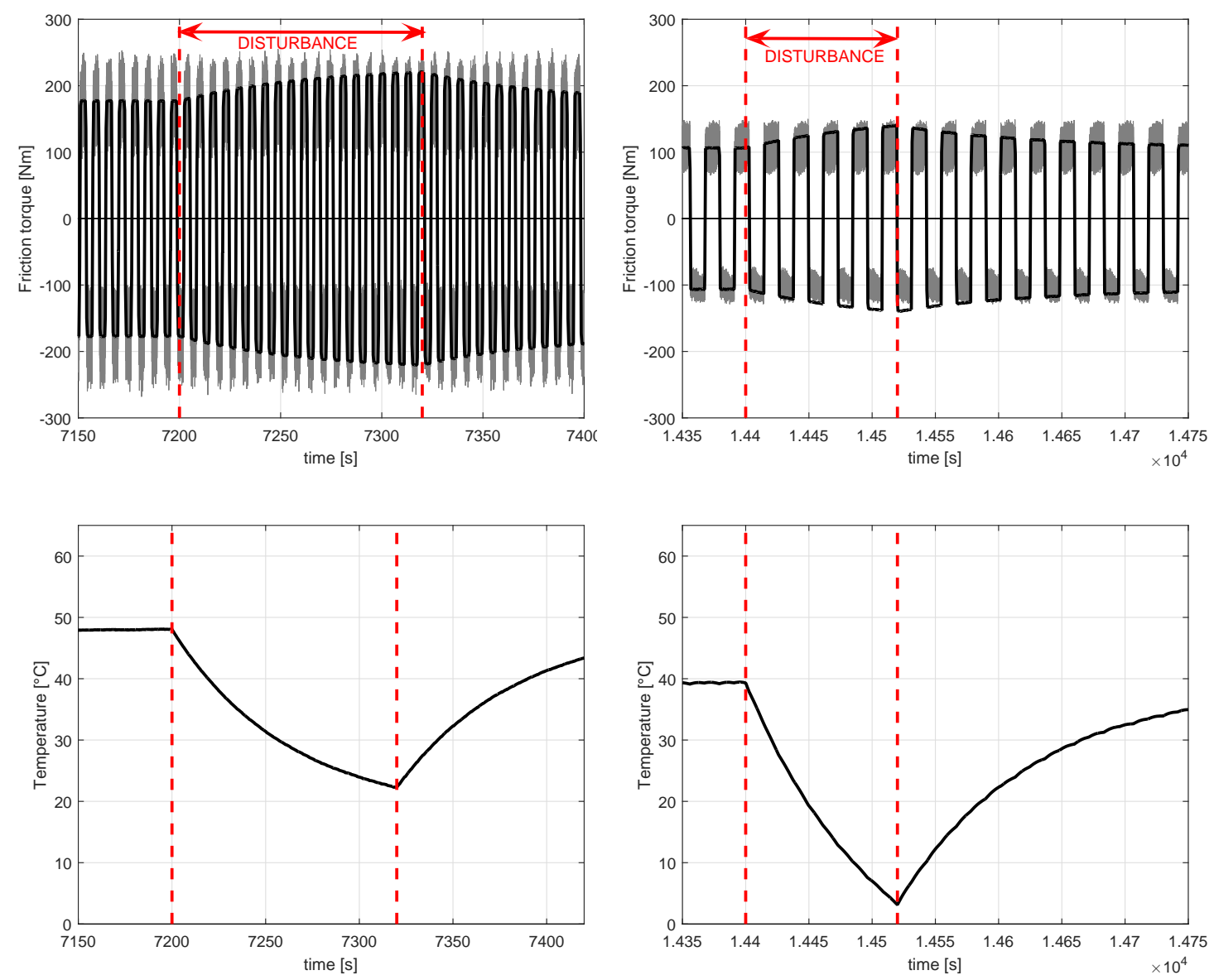

Figure 19: Friction torque obtained from experimental data (grey thin line) and estimated one (black thick line) for joint 1 when a constant torque disturbance has been applied for 120 seconds after 2 (left) and 4 (right) hours respectively, and related temperature.

\section{Conclusions}

In this paper a polynomial friction model for industrial robot manipulators that takes into account temperature effects has been proposed. The model can be applied without a measurement of the joint internal temperature. A simple identification procedure can be employed to estimate the model parameters and then an observer based technique can be suitably applied to compensate for a wrong estimation of the initial conditions (that is, of the initial joint temperature). Experimental results have been shown in order to demonstrate the effectiveness of the method. Future works will consist of applying temperature extension to more complex friction phenomena descriptions, such as Stribeck or LuGre models, that can be relevant for some kind of specific robot operations where slow transitions through zero velocity are required. Furthermore, it will be investigated the use of the proposed model for long term maintenance analyses. 


\section{Acknowledgements}

This work is partially developed within the European Union H2020 program under grant agreement n. 637095 (FourByThree) and ECSEL-2016-1 under grant agreement n. 737453 (I-MECH).

\section{References}

[1] K. J. Åström and C. Canudas de Wit. Revisiting the lugre model. IEEE Control Systems Magazine, 28(6):101-114, 2008.

[2] K. J. Åström, C. Canudas de Wit, H. Olsson, and P. Lischinsky. A new model for control of systems with friction. IEEE Transaction on Automatic Control, 40(3):419-425, 1995.

[3] K. J. Åström, C. Canudas de Wit, H. Olsson, P. Lischinsky, and P. Gäfvert. Friction models and friction compensation. European Journal of Control, 4(3):176-195, 1998.

[4] A. Carvalho Bittencourt and P. Axelsson. Modeling and experiment design for identification of wear in a robot joint under load and temperature uncertainties based on friction data. IEEE/ASME Transactions on Mechatronics, 19(5):1694-1706, 2014.

[5] A. Carvalho Bittencourt and S. Gunnarsson. Static friction in a robot joint-modeling and identification of load and temperature effects. Journal of Dynamic Systems, Measurement, and Control, 134(5), 2012.

[6] A. Carvalho Bittencourt, E. Wernholt, S. Sander-Tavallaey, and T. Brogardh. An extended friction model to capture load and temperature effects in robot joints. In Proceedings IEEE/RSJ International Conference on Intelligent Robots and Systems, pages 6161-6167, Taipei (Taiwan), 2010.

[7] B. Bona and M. Indri. Friction compensation in robotics: an overview. In Proceedings of the 44th IEEE Conference on Decision and Control and the European Control Conference, pages 4360-4367, Seville (E), 2005.

[8] F. B. Carlson, A. Robertsson, and R. Johansson. Modeling and identification of position and temperature dependent friction phenomena without temperature sensing. In Proceedings of the IEEE/RSJ International Conference on Intelligent Robots and Systems, pages 3045-3051, Hamburg (D), 2015.

[9] P. R. Dahl. A solid friction model. Technical Report ADA041920, Space and Missile Systems Organization, Air Force System Command, Los Angeles, CA (USA), 1968.

[10] H. Dankowicz. On the modeling of dynamic friction phenomena. ZAMM - Journal of Applied Mathematics and Mechanics, 79(6):399-409, 1999.

[11] G. Ferretti, G. Magnani, and P. Rocco. Single and multistage integral friction models. IEEE Transactions on Automatic Control, 49(12):2292-2297, 2004.

[12] L. Freidovich, A. Robertsson, A. Shiriaev, and R. Johansson. Lugre-model-based friction compensation. IEEE Transactions on Control Systems Technology, 18(1):194-200, 2010. 
[13] S. S. Ge, T. H. Lee, and S. X. Ren. Adaptive friction compensation of servo mechanisms. International Journal of Systems Science, 32:523-532, 2001.

[14] V. I. Gervini, E. M. Hemerly, and S. C. P. Gomes. Active control of flexible one-link manipulators using wavelet networks. Robotica, 31:1275-1283, 2013.

[15] S. I. Han, K. S. Lee, M. G. Park, and J. M. Lee. Robust adaptive deadzone and friction compensation of robot manipulator using RWCMAC network. Journal of Mechanical Science and Technology, 25:15831594, 2011.

[16] A. Janot, P.-O. Vandanjon, and M. Gautier. A generic instrumental variable approach for industrial robot identification. IEEE Transactions on Control Systems Technology, 22(1):132-145, 2014.

[17] F. Jatta, G. Legnani, and A. Visioli. Friction compensation in hybrid force/velocity control of industrial manipulators. IEEE Transactions on Industrial Electronics, 53(2):604-613, 2006.

[18] F. E. Kennedy. Frictional heating and contact temperatures. In B. Bhushan, editor, Modern Tribology Handbook, chapter 6. CRC Press, Boca Raton (FL), 2001.

[19] L. Lampaert, J. Swevers, and F. Al-Bender. An integrated friction model structure with improved presliding behaviour for accurate friction compensation. IEEE Transactions on Automatic Control, 40:675-686, 2000.

[20] V. Lampaert, F. Al-Bender, and J. Swevers. A generalized maxwell-slip friction model appropriate for control purposes. In Proceedings of IEEE International Conference on Physics and Control, volume 4, pages 1170-1177, St. Petersburg (Russia), 2003.

[21] L. Márton and F. van der Linden. Temperature dependent friction estimation: Application to lubricant health monitoring. Mechatronics, 22:1078-1084, 2012.

[22] M. Neubauer, H. Gattringer, and H. Bremer. A persistent method for parameter identification of a sevenaxes manipulator. Robotica, 33:1099-1112, 2015.

[23] T. Piatkovsky. Dahl and lugre dynamic friction models - the analysis of selected properties. Mechanism and Machine Theory, 73:91-100, 2014.

[24] T. Piatkovsky. Gms friction model approximation. Mechanism and Machine Theory, 75:1-11, 2014.

[25] D.M. Pirro, M. Webster, and E. Daschner. Lubrication Fundamentals, Revised and Expanded. CRC Press, 2016.

[26] M. Ruderman. Presliding hysteresis damping of lugre and maxwell-slip friction models. Mechatronics, 30:225-230, 2015.

[27] W. Sanxiu and J. Shengtao. Adaptive friction compensation of robot manipulator. In Electronics and Signal Processing (W. Hu editor), pages 127-134, 2011. 
[28] L. Simoni, M. Beschi, G. Legnani, and A. Visioli. Friction modeling with temperature effects for industrial robot manipulators. In Proceedings of the IEEE/RSJ International Conference on Intelligent Robots and Systems, pages 3524-3529, Hamburg (D), 2015.

[29] A. Stolt, F. B. Carlson, M. M. Ghazaei Ardakani, I. Lundberg, A. Robertsson, and R. Johansson. Sensorless friction-compensated passive lead-through programming for industrial robots. In Proceedings of the IEEE/RSJ International Conference on Intelligent Robots and Systems, pages 3530-3537, Hamburg (D), 2015.

[30] Y.-H. Sun, T. Chen, C. Q. Wu, and C. Shafai. A comprehensive experimental setup for identification of friction model parameters. Mechanism and Machine Theory, 100:338-357, 2016.

[31] J. Swevers, F. Al-Bender, C. G. Ganseman, and T. Prajogo. Modification of the leuven integrated friction model structure friction models. IEEE Transactions on Automatic Control, 47:683-687, 2002.

[32] V. van Geffen. A study of friction models and friction compensation. Technical Report DCT 2009.118, Technische Universiteit Eindhoven, Department Mechanical Engineering, Dynamics and Control Technology Group, Eindhoven (NL), 2009.

[33] E. Villagrossi, G. Legnani, N. Pedrocchi, F. Vicentini, L. M. Tosatti, F. Abbà, and A. Bottero. Robot dynamic model identification through excitation trajectories minimizing the correlation influence among essential parameters. In Proceedings of the 11th IEEE Conference on Informatics in Control, Automation and Robotics, pages 475-482, Vienna (A), 2014.

[34] E. Villagrossi, N. Pedrocchi, F. Vicentini, and L. Molinari Tosatti. Optimal robot dynamics local identification using genetic-based path planning in workspace subregions. In Proceedings of the IEEE/ASME International Conference on Advanced Intelligent Mechatronics, pages 932-937, Wollongong (AUS), 2013.

[35] A. Visioli and G. Legnani. On the trajectory tracking control of industrial SCARA robot manipulators. IEEE Transactions on Industrial Electronics, 49(1):224-232, 2002.

[36] J. Y. Yoon and D. L. Trumper. Friction modeling, identification, and compensation based on friction hysteresis and dahl resonance. Mechatronics, 24:734-741, 2014.

[37] Z. Zhongyu. The study of friction variation with temperature in a harmonic drive system - modeling and control. Master's thesis, Concordia University, Montreal (CA), 2006. 